Research Article

\title{
Transportation Inequalities for Coupled Fractional Stochastic Evolution Equations Driven by Fractional Brownian Motion
}

\author{
Wentao Zhan, Yuanyuan Jing, Liping Xu $(D)$, and Zhi Li \\ School of Information and Mathematics, Yangtze University, Jingzhou 434023, China \\ Correspondence should be addressed to Zhi Li; lizhi_csu@126.com
}

Received 27 September 2019; Accepted 13 February 2020; Published 7 May 2020

Academic Editor: Nickolai Kosmatov

Copyright (C) 2020 Wentao Zhan et al. This is an open access article distributed under the Creative Commons Attribution License, which permits unrestricted use, distribution, and reproduction in any medium, provided the original work is properly cited.

In this paper, we consider the existence and uniqueness of the mild solution for a class of coupled fractional stochastic evolution equations driven by the fractional Brownian motion with the Hurst parameter $H \in(1 / 4,1 / 2)$. Our approach is based on Perov's fixed-point theorem. Furthermore, we establish the transportation inequalities, with respect to the uniform distance, for the law of the mild solution.

\section{Introduction}

In the research of various fields of science and engineering, fractional stochastic differential equations (SDEs) play a significant role in the modeling of many complex phenomena in diverse areas. The intensive development in both theory and applications of fractional SDEs was investigated in [1-4]. In addition, many scholars have also developed interest in systems with memory or after effect (i.e., systems with finite delays in the state equation). Therefore, it is necessary to study stochastic evolution equations with finite delays. The equation is widely used in network flow analysis, mathematical finance, astrophysics, hydrology, image processing, and other directions $[5,6]$. For the nature of the existence and uniqueness of the mild solutions, Sakthivel et al. [7] considered the nonlinear-type fractional SDE, Li [8] established stochastic delay fractional evolution equations driven by fractional Brownian motion in a Hilbert space, and Mophou [9] was concerned about impulsive fractional semilinear differential equations.

Moreover, under the research of many scholars, transportation inequalities are developed greatly in various SDEs and stochastic systems with respect to the different measure conditions. Among others, the Girsanov transformation argument introduced in [10] has been efficiently applied, e.g., Wu and Zhang [11] considered infinite-dimensional dynamical systems with respect to $L^{2}$ metric; Üstünel [12] studied the multivalued SDE and singular SDE under uniform distance; besides, Bao et al. [13] investigated the neutral functional SDE with respect to both the uniform distance and the $L^{2}$ distance; Saussereau [14] researched the SDE driven by a fractional Brownian motion; futhermore, Li and Luo [15] took it into account that stochastic delay evolution equations driven by fractional Brownian motion with the hurst parameter $H>1 / 2$ under the $L^{2}$ metric and the uniform metric; and Boufoussi and Hajji [16] established the transportation inequalities, with respect to the uniform distance, for the law of the mild solution for a neutral stochastic differential equation with finite delay, driven by a fractional Brownian motion with the Hurst parameter lesser than $1 / 2$ in a Hilbert space.

In connection with the aforementioned works, in this paper, we investigate the existence, uniqueness, and property $T_{2}(C)$ under the uniform distance for the law of mild solution of the coupled fractional stochastic delay evolution equations with finite delay driven by a fractional Brownian motion with the Hurst parameter $0<H<1 / 2$ : 


$$
\begin{cases}{ }^{c} D^{\alpha_{1}}(x(t))=\left(A_{1} x(t)+f_{1}\left(t, x_{t}, y_{t}\right)\right) \mathrm{d} t+\sigma_{1}(t) \mathrm{d} B_{1}^{H}(t), & t \in[0, T], \\ { }^{c} D^{\alpha_{2}}(y(t))=\left(A_{2} y(t)+f_{2}\left(t, x_{t}, y_{t}\right)\right) \mathrm{d} t+\sigma_{2}(t) \mathrm{d} B_{2}^{H}(t), & t \in[0, T], \\ x(t)=\phi_{1}(t), & t \in[-r, 0], \\ y(t)=\phi_{2}(t), & t \in[-r, 0],\end{cases}
$$

where ${ }^{c} D^{\alpha_{i}}$ is the Caputo fractional derivative of order $\alpha_{i} \in(1 / 2,1]$, for each $i=1,2$, as for the state $x(\cdot), y(\cdot)$ has values in a real and separable Hilbert $X$ with an inner product $(\cdot, \cdot)_{X}$ and norm $\|\cdot\|_{X}$, where $\left\{A_{i}, i=1,2\right\}$ are the infinitesimal generators of analytic semigroups of bounded linear operators $\left\{T_{i}(t), t \geq 0\right\}, B_{i}^{H}$ is the fractional Brownian motion on a real and separable Hilbert space $Y$, with the Hurst parameter $H \in(0,1 / 2)$, and let $r>0$ denote the constant. As for $y_{t}$, we mean the segment solution which is defined in the usual way, that is, if $y(\cdot, \cdot):[-r, T] \times \Omega \longrightarrow X$, then for any $t>0$

$$
y_{t}(\theta, \omega)=y(t+\theta, \omega), \quad \theta \in[-r, 0], \omega \in \Omega .
$$

Before describing the properties fulfilled by operators $f_{i}, \sigma_{i}$, we need to introduce some nontations and describe some spaces. Let $\mathscr{D}_{0}$ denote the space of all continuous functions $\varphi:[-r, 0] \times \Omega \longrightarrow X$ such that $\varphi(\theta, \cdot)$ is $\mathscr{F}_{0}$-measurable for each $\theta \in[-r, 0]$ and $\int_{-r}^{0} \mathbb{E}\|\varphi\|_{X}^{2} \mathrm{~d} t<\infty$. In the space $\mathscr{D}_{0}$, we endow with the following norm:

$$
\|\varphi\|_{D_{0}}^{2}=\int_{-r}^{0} \mathbb{E}\|\varphi(t)\|_{X}^{2} \mathrm{~d} t .
$$

Next, we denote by $C\left(a, b ; L^{2}(\Omega ; X)\right)=C\left(a, b ; L^{2}(\Omega\right.$, $\mathscr{F}, \mathbb{P} ; \mathrm{X}))$ the Banach space of all continuous functions from $[a, b]$ into $L^{2}(\Omega ; X)$. Now, fixing $T>0$, we define

$$
\begin{aligned}
\mathscr{D}_{T}= & \left\{y: y \in C\left(-r, T ; L^{2}(\Omega ; X)\right), \sup _{t \in[0, T]} \mathbb{E}\left(\|y(t)\|_{X}^{2}\right)<\infty,\right. \\
& \left.\int_{-r}^{0} \mathbb{E}\|y(t)\|_{X}^{2} \mathrm{~d} t<\infty\right\},
\end{aligned}
$$

endowing with the following norm:

$$
\|y\|_{\mathscr{D}_{T}}=\sup _{t \in[0, T]} \sqrt{\mathbb{E}\left(\|y(t)\|_{X}^{2}\right)}+\|y(t)\|_{\mathscr{D}_{0}} .
$$

We give initial data $\varphi_{1}, \varphi_{2} \in \mathscr{D}_{0}$, and $Y$ is another real and separable Hilbert space, and $B_{Q_{i}}^{H}=B_{i}^{H}$ is a $Y$-valued fractional Brownian motion with increment covariance given by a nonnegative trace class operator $Q_{i}$, and $L(Y, X)$ represents the space of all bounded, continuous, and linear operators from $Y$ into $X$.

We denote $f_{i}: J \times D_{0} \times D_{0} \longrightarrow X$ and $\sigma_{i}$ : $J \longrightarrow L_{Q_{i}}^{0}(Y, X)$. Here, let us denote $L_{Q_{i}}^{0}(Y, X)$ by the space of all $Q_{i}$-Hilbert-Schmidt operators from $Y$ into $X$ for each $i=1,2$ which will also be introduced in the next section.

Now, let us present the relevant knowledge of transportation inequalities. To connect the measure distances with the probability measures, we consider the transportation distance, also called as Wasserstein distance. Let $(E, d)$ be a metric space provided with the $\sigma$-field $\mathscr{B}$, such that $\mathrm{d}(\cdot, \cdot)$ is $\mathscr{B} \times \mathscr{B}$-measurable. Fixing $p \geq 1$ and for any probability measures $\mu$ and $\nu$ on $E$, we define the Wasserstein distance of order $p$ between $\mu$ and $v$ as

$$
W_{p}^{d}(\mu, v)=\inf _{\pi \in \prod(\mu, v)}\left(\int_{\mathrm{E} \times \mathrm{E}} \mathrm{d}(x, y)^{p} \mathrm{~d} \pi(x, y)\right)^{(1 / p)},
$$

where $\Pi(\mu, \nu)$ denotes the totality of probability measures on $E \times E$ with the marginal $\mu$ and $\nu$. The relative entropy of $\nu$ with respect to $\mu$ is defined as

$$
H(v, \mu)= \begin{cases}\int \log \frac{\mathrm{d} \nu}{\mathrm{d} \mu} \mathrm{d} v, & \nu \ll \mu, \\ +\infty, & \text { otherwise. }\end{cases}
$$

The probability measure $\mu$ satisfies $L^{p}$-transportation inequality on $(E, d)$ if there exists a constant $C \geq 0$ such that for any probability measure $v$,

$$
W_{p}^{d}(\mu, \nu) \leq \sqrt{2 C H(\nu \mid \mu)}
$$

As usual, we write $\mu \in T_{p}(C)$ for this relation. The property $T_{2}(C)$ is of particular interest. We will investigate the property $T_{2}(C)$ for the law of the mild solution of stochastic delay evolution equations driven by fractional Brownian motion with the Hurst parameter $1 / 4<H<1 / 2$ under the uniform distance.

This paper is organized as follows. In Section 2, we introduce some preliminaries used in this paper such as stochastic calculus, some properties of generalized Banach spaces, and fractional calculus. In Section 3, we state and prove the existence and uniqueness of the mild solution by using Perov's fixed-point type in generalized Banach spaces. In Section 4, we investigate the property $T_{2}(C)$ for the law of the solution of fractional stochastic delay evolution equations driven by fractional Brownian motion with the Hurst parameter $1 / 4<H<1 / 2$ under the uniform metric. In Section 5 , we present an example to illustrate the efficiency of the obtained result.

\section{Preliminaries}

In this section, we introduce some notations and recall definitions and preliminary results which are used throughout this paper.

Let $\left(\Omega, \mathscr{F}, \mathbb{P},\left\{\mathscr{F}_{t}\right\}_{t>0}\right)$ be a complete probability space furnished with a normal filtration $\left\{\mathscr{F}_{t}\right\}_{t \geq 0}$. We postulate that the operator $A_{i}$ is self-adjoint and there exists the eigenvectors $e_{k}$ corresponding to eigenvalues $\gamma_{k}$ such that 


$$
A_{i} e_{k}=\gamma_{k} e_{k}, e_{k}=\sqrt{2} \sin (k \pi), \quad \gamma_{k}=\pi^{2} k^{2}, k \in \mathbb{N}^{+} .
$$

For each $\sigma>0, A_{i}^{(\sigma / 2)} e_{k}=\gamma_{k}^{(\sigma / 2)}, k=1,2,3, \ldots$, and let $\dot{H}^{\sigma}$ be the domain of $\left\{A_{i}^{(\sigma / 2)}\right\}$ defined by

$$
\dot{H}^{\sigma}=D\left(A_{i}^{(\sigma / 2)}\right)=\left\{v \in L^{2}(X), \quad \text { s.t. } \quad\|v\|_{\dot{H}^{\sigma}}^{2}=\sum_{k=0}^{\infty} \gamma_{k}^{(\sigma / 2)} v_{k}^{2}<\infty\right\} \text {, }
$$

where the vector $v_{k}=\left(v, e_{k}\right)$ and the norm $\|v\|_{\dot{H}^{\sigma}}=\left\|A_{i}^{(\sigma / 2)} v\right\|$. Let $L^{2}(X)$ be an $X$-valued Hilbert space with the inner product $\mathbb{E}(\cdot, \cdot)$ and norm $\mathbb{E}\|\cdot\|$, and it is given by

$$
L^{2}(X)=\left\{\chi: \mathbb{E}\|\chi\|_{X}^{2}=\int_{\Omega}\|\chi(\omega)\|_{X}^{2} \mathrm{~d} \mathbb{P}(\omega)<\infty, \quad \omega \in \Omega\right\} .
$$

Definition 1. For $H \in(0,1)$, a continuous centered Gaussian process $\left\{B^{H}(t)\right\}_{t \in[0, \infty)}$ with covariance function

$$
\begin{aligned}
R_{H}(t, s) & =\mathbb{E}\left[B^{H}(t) B^{H}(s)\right] \\
& =\frac{1}{2}\left(t^{2 H}+s^{2 H}-|t-s|^{2 H}\right), \quad t, s \in[0, \infty),
\end{aligned}
$$

is called a one-dimensional fractional Brownian motion ( $\mathrm{fBm})$, and $\mathrm{H}$ is the Hurst parameter. In particular, when $H=1 / 2, B^{H}(t)$ represents a standard Brownian motion.

Now, let us aim at the Wiener integral with respect to the $\mathrm{fBm}$. To begin with, $B^{H}(t)$ has following integral expression (see [17]):

$$
B^{H}(t)=\int_{0}^{t} K_{H}(t, s) \mathrm{d} B(s),
$$

where $B=\{B(t): t \in[0, T]\}$ is a Wiener process, $K_{H}(t, s)$ is a square integrable kernel, for $0<H<1 / 2$, and $t>s$; the formula is as follows (see [17]):

$$
K_{H}(t, s)=c_{H}\left[\left(\frac{t}{s}\right)^{H-(1 / 2)}(t-s)^{H-(1 / 2)}-\left(H-\frac{1}{2}\right) s^{(1 / 2)-H} \int_{s}^{t}(u-s)^{H-(1 / 2)} u^{H-(3 / 2)} \mathrm{d} u\right]
$$

where $c_{H}=\sqrt{H /((1-H) \beta(1-2 H, H+1 / 2))}$ and $\beta(\cdot, \cdot)$ denotes the Beta function. $K_{H}(t, s)=0, t \leq s$. Since $0<H<1 / 2$, from (14), we can infer that

$$
\begin{aligned}
\left|K_{H}(t, s)\right| & \leq c_{H}\left[(t-s)^{H-(1 / 2)}+\left(\frac{1}{2}-H\right) s^{(1 / 2)-H} \int_{s}^{t}(u-s)^{H-(1 / 2)} u^{-H-(1 / 2)} u^{2 H-1} \mathrm{~d} u\right] \\
& \leq c_{H}\left[(t-s)^{H-(1 / 2)}+\left(\frac{1}{2}-H\right) s^{(1 / 2)-H} \int_{s}^{t}(u-s)^{H-(1 / 2)} u^{-H-(1 / 2)} \mathrm{d} u\right] .
\end{aligned}
$$

Then, we obtain

$$
\left|K_{H}(t, s)\right| \leq 2 c_{H}\left((t-s)^{H-(1 / 2)}+s^{H-(1 / 2)}\right) .
$$

Taking the derivative of (14) with respect to $t$, we can have

$$
\frac{\partial K_{H}}{\partial t}(t, s)=c_{H}(H-(1 / 2))\left(\frac{t}{s}\right)^{H-(1 / 2)}(t-s)^{H-(3 / 2)} .
$$

Apparently, we can obtain the following inequality:

$$
\left|\frac{\partial K_{H}}{\partial t}(t, s)\right|=c_{H}\left(\frac{1}{2}-H\right)(t-s)^{H-(3 / 2)} .
$$

Let $\mathscr{H}$ be the Hilbert space defined as the closure of the vector space spanned by the set of step functions $\left\{\mathbf{I}_{[0, t]}, t \in[0, T]\right\}$ with respect to the scalar product:

$$
\left\langle\mathbf{I}_{[0, t]}, \mathbf{I}_{[0, s]}\right\rangle=R_{H}(t, s), \quad \forall t, s \in[0, T] .
$$

Now, we consider the operator $K_{H, T}^{*}$ from $\mathscr{H}$ to $L^{2}([0, T])$ defined by

$$
\left(K_{H, T}^{*} \varphi\right)(s)=K_{H}(T, s) \varphi(s)+\int_{s}^{T}(\varphi(r)-\varphi(s)) \frac{\partial K_{H}}{\partial r}(r, s) \mathrm{d} r .
$$

Furthermore, $K_{H, T}^{*}$ is an isometry between $\mathscr{H}$ and $L^{2}([0, T])$ (see $\left.[18]\right)$. Taking account for $B=\{B(t)$, $t \in[0, T]\}$ defined by

$$
B(t)=B^{H}\left(\left(K_{H}^{*}\right)^{-1} \mathrm{I}_{[0, t]}\right),
$$

it turns out that $B$ is a Wiener process. Moreover, for any $\varphi \in \mathscr{H}$, with (13), we have

$$
\int_{0}^{T} \varphi(S) \mathrm{d} B^{H}(s):=B^{H}(\varphi)=\int_{0}^{T}\left(K_{H, T^{\varphi}}^{*}\right)(t) \mathrm{d} B(t) .
$$

For any $0 \leq t \leq T$, we can also deduce

$$
\begin{aligned}
\int_{0}^{t} \varphi(s) \mathrm{d} B^{H}(s) & :=\int_{0}^{T}\left(K_{H, T}^{*} \varphi \mathrm{I}_{[0, t)}\right)(s) \mathrm{d} B(s) \\
& =\int_{0}^{t}\left(K_{H, t}^{*} \varphi\right)(s) \mathrm{d} B(s),
\end{aligned}
$$


where $K_{H, t}^{*}$ is defined in the same way as in (20) with $t$ instead of $T$. Next, we will use the notation $K_{H, t}^{*}$ without specifying the parameter $t \in[0, T]$.

Let $\left(X,\|\cdot\|_{X},\langle\cdot, \cdot\rangle_{X}\right)$ and $\left(Y,\|\cdot\|_{Y},\langle\cdot, \cdot\rangle_{Y}\right)$ be two real, separable Hilbert spaces and let $\mathscr{L}(Y, X)$ denote the space of all bounded linear operators from $Y$ to $X$. Let $Q \in \mathscr{L}(Y, X)$ be a nonnegative self-adjoint operator i.e., $Q e_{n}=\lambda_{n} e_{n}$ with trace $\operatorname{tr} Q=\sum_{n=1}^{\infty} \lambda_{n}<\infty$, where $\lambda_{n} \in \mathbb{R}^{+}$and $\left\{e_{n}\right\}_{n \geq 1}$ is a complete orthonormal basis in $Y$. We define the infinitedimensional $\mathrm{fBm}$ on $Y$ with covariance $Q$ by the following formula:

$$
B_{Q}^{H}(t)=\sum_{n=1}^{\infty} \sqrt{\lambda_{n}} e_{n} B_{n}^{H}(t), \quad t \geq 0,
$$

where $\left\{B_{n}^{H}(t)\right\}_{n \in \mathbb{N}}$ be a sequence of one-dimensional mutually independent standard fractional Brownian motions on $(\Omega, \mathscr{F}, \mathbb{P}) . B_{n}^{H}(t)$ is a $Y$-valued Gaussian process, starting from 0 , and has zero mean and covariance:

$$
\begin{array}{r}
\mathbb{E}\left\langle B_{Q}^{H}(t), x\right\rangle\left\langle B_{Q}^{H}(s), y\right\rangle=R(s, t)\langle Q(x), y\rangle, \\
\text { for all } x, y \in Y \text { and } t, s \in[0, T] .
\end{array}
$$

Let $\mathscr{L}_{2}^{0}(Y, X)$ be the space of all $\xi \in \mathscr{L}(Y, X)$ such that $\xi Q^{(1 / 2)}$ is a Hilbert-Schmidt operator. The norm is given by

$$
\|\xi\|_{L_{2}^{0}(Y, X)}^{2}:=\sum_{n=1}^{\infty}\left\|\sqrt{\lambda_{n} \xi(s) e_{n}}\right\|^{2}=\operatorname{tr}\left(\xi Q \xi^{*}\right)<\infty .
$$
$X$.

Then, $\xi$ is called a $Q$-Hilbert-Schmidt operator from $Y$ to

Definition 2. Let $\varphi:[0, T] \longrightarrow \mathscr{L}_{2}^{0}(Y, X)$. Then, the Wiener integral of $\varphi$ with respect to the $\mathrm{fBm} B_{Q}^{H}$ is defined as follows:

$$
\begin{aligned}
\int_{0}^{t} \varphi(s) \mathrm{d} B_{Q}^{H}(s) & :=\sum_{n=1}^{\infty} \int_{0}^{t} \sqrt{\lambda_{n}} \varphi(s) e_{n} \mathrm{~d} B_{n}^{H}(s) \\
& =\sum_{n=1}^{\infty} \int_{0}^{t} \sqrt{\lambda_{n}}\left(K_{H}^{*}\left(\varphi e_{n}\right)\right)(s) \mathrm{d} B_{n}(s),
\end{aligned}
$$

where $B_{n}$ is the standard Brownian motion used to represent $B_{n}^{H}$ as in (13), and the sum above is finite when $\sum_{n=1}^{\infty} \lambda_{n}\left\|K_{H}^{*}\left(\varphi e_{n}\right)\right\|<\infty$.

The classical Banach contraction principle was extended for contractive maps on spaces endowed with a vector-valued metric space by Perov [19] in 1964 and Precup $[20,21]$. Now, we recall some useful definitions and results.

Definition 3. Let $Z$ be a nonempty set. We denote by a vector-valued metric on $Z$ defined as a mapping $d: Z \times$ $Z \longrightarrow \mathbb{R}^{n}$ with the following properties:

(1) $\mathrm{d}(u, v) \geq 0$ for all $u, v \in Z ; \mathrm{d}(u, v)=0$, only if $u=v$.

(2) $\mathrm{d}(u, v)=\mathrm{d}(u, v)$ for all $u, v \in Z$.

(3) $\mathrm{d}(u, v) \leq \mathrm{d}(u, w)+\mathrm{d}(w, v)$ for all $u, v, w \in Z$.
Now, we consider a generalized metric space $(Z, d)$. For $r=\left(r_{1}, \ldots, r_{n}\right) \in \mathbb{R}_{+}^{n}$, we will define the open ball centered in $x_{0}$ with radius $r$ :

$$
B\left(x_{0}, r\right)=\left\{x \in Z: \mathrm{d}\left(x_{0}, x\right)<r\right\},
$$

and the closed ball centered in $x_{0}$ with radius $r$ :

$$
\overline{B\left(x_{0}, r\right)}=\left\{x \in Z: \mathrm{d}\left(x_{0}, x\right) \leq r\right\} .
$$

We state that for a generalized metric space, the notation of open and closed sets, convergence, Cauchy sequence, and completeness in a generalized metric space are similar to those in usual metric spaces. If $x, y \in \mathbb{R}^{n}, x=\left(x_{1}, \ldots, x_{n}\right)$, $y=\left(y_{1}, \ldots, y_{n}\right)$, by $x \leq y$ then we mean $x_{i} \leq y_{i}, i=1, \ldots, n$. Also, $|x|=\left(\left|x_{1}\right|, \ldots,\left|x_{n}\right|\right)$ and $\max (x, y)=\max \left(\max \left(x_{1}\right.\right.$, $\left.\left.y_{1}\right), \ldots, \max \left(x_{n}, y_{n}\right)\right)$. If $c \in \mathbb{R}$, then $x \leq c$ means $x_{i} \leq c$ for each $i=1, \ldots, n$.

Definition 4. A generalized metric space $(Z, d)$ where $\mathrm{d}(x, y):=\left(\begin{array}{c}\mathrm{d}_{1}(x, y) \\ \vdots \\ \mathrm{d}_{n}(x, y)\end{array}\right)$ is complete, if for every $i=1, \ldots, n,\left(Z, d_{i}\right)$ is a complete metric space.

Definition 5. We denote that a real square matrix $M$ is convergent to zero if and only if its spectral radius $\rho(M)$ is strictly less than 1 . In other words, it means that all the eigenvalues of $M$ are in the open unit disc (i.e., $\quad|\lambda|<1$, for every $\lambda \in \mathbb{C}$ with $\operatorname{det}(M-\lambda I)=0$, where $I$ denotes the unit matrix of $\left.\mathscr{M}_{n \times n}(\mathbb{R})\right)$.

Definition 6. We denote that a nonsingular matrix $A=\left(a_{i j}\right)_{1 \leq i, j \leq n} \in \mathscr{M}_{n \times n}(\mathbb{R})$ has the absolute value property if

$$
A^{-1}|A| \leq I \text {, }
$$

where

$$
|A|=\left(\left|a_{i j}\right|\right)_{1 \leq i, j \leq n} \in \mathscr{M}_{n \times n}(\mathbb{R}) .
$$

Now, we need to use the following fixed-point theorem to prove the existence and uniqueness of mild solution for (1).

Theorem 1 (see [19]). Let $(Z, d)$ be a complete generalized metric space with $d: Z \times Z \longrightarrow \mathbb{R}^{n}$ and let operator $N: Z \longrightarrow Z$ be such that

$$
\mathrm{d}(N(x), N(y)) \leq M \mathrm{~d}(x, y),
$$

for all $x, y \in Z$ and some nonnegative square matrix $M$. If the matrix $M$ is convergent to 0 , that is, $M^{k} \longrightarrow 0$ as $k \longrightarrow 0$, then operator $N$ has a unique fixed point $x_{*} \in Z$ :

$$
\mathrm{d}\left(N^{k}\left(x_{0}, x_{*}\right)\right) \leq M^{k}(I-M)^{-1} \mathrm{~d}\left(N\left(x_{0}, x_{0}\right)\right),
$$

for every $x_{0} \in Z$ and $k \geq 1$. 
Definition 7. The fractional integral of index $\alpha$ with the lower limit 0 for a function $f$ can be written as

$$
I^{\alpha} f(t)=\frac{1}{\Gamma(\alpha)} \int_{0}^{t} \frac{f(s)}{(t-s)^{1-\alpha}} \mathrm{d} s, \quad t>0, \alpha>0,
$$

provided the right-hand side is pointwise defined on $[0,+\infty)$, where $\Gamma$ is the gamma function, which is defined by $\Gamma(y):=\int_{0}^{\infty} t^{y-1} e^{-t} \mathrm{~d} t$.

Definition 8. The Caputo derivative of index $\alpha$ for a function $f \in C^{n}([0, \infty))$ is defined as

$$
\begin{array}{r}
{ }^{C} D_{t}^{\alpha} f(t)=\frac{1}{\Gamma(n-\alpha)} \int_{0}^{t} \frac{f^{(n)}(s)}{(t-s)^{\alpha+1-n}} \mathrm{~d} s=I^{n-\alpha} f^{(n)}(t), \\
t>0, n-1<\alpha<n .
\end{array}
$$

\section{Existence and Uniqueness}

In this section, we investigate the existence and uniqueness of a mild solution for (1). First of all, we will give some hypotheses which will be used to prove our main result; for this question, we assume that the following conditions hold.
$(\mathscr{H} .1)$ There exists constants $a_{f_{i}}, b_{f_{i}} \in \mathbb{R}^{+}$for each $i=$ $1,2, \ldots$ such that

$$
\begin{aligned}
& \int_{0}^{t}\left\|f_{i}\left(s, x_{s}, y_{s}\right)-f_{i}\left(s, \bar{x}_{s}, \bar{y}_{s}\right)\right\|_{X}^{2} \\
& \quad \leq a_{f_{i}} \int_{-r}^{t}\|x(s)-\bar{x}(s)\|_{X}^{2} \mathrm{~d} s+b_{f_{i}} \int_{-r}^{t}\|y(s)-\bar{y}(s)\|_{X}^{2} \mathrm{~d} s, \\
& \quad \text { for all } x, y, \bar{x}, \bar{y} \in C([-r, T] ; X) .
\end{aligned}
$$

( $\mathscr{H} .2)$ The function $\sigma:[0, T] \longrightarrow L_{Q}^{0}(Y, X)$ satisfies the following Hölder continuous conditions, that is, there exists a constant $C_{\sigma}>0$ such that for all $t, s \in[0, T]$,

$$
\|\sigma(t)-\sigma(s)\|_{\mathscr{L}_{\mathrm{Q}}^{0}} \leq C_{\sigma}|t-s|^{\gamma},
$$

where $\gamma>1-2 H$.

Now, we state the following definition of mild solution for our problem.

Definition 9. A $\mathscr{H}$-valued process $u(t)=(x(t), y(t))$ is called a mild solution of (1) with respect to the probability space $(\Omega, \mathscr{F}, \mathbb{P}), \quad$ if $\quad x, y \in C\left(-r, T ; L^{2}(\Omega ; X)\right), \quad(x(t), y(t))=$ $\left(\phi_{1}(t), \phi_{2}(t)\right)$ for $t \in[-r, 0]$, and for each $t \in[0, T]=J, u(t)$ it satisfies the following integral equation:

$$
\left\{\begin{array}{l}
x(t)=T_{\alpha_{1}} \phi_{1}(0)+\int_{0}^{t}(t-s)^{\alpha_{1}-1} E_{\alpha_{1}}(t-s) f_{1}\left(s, x_{s}, y_{s}\right) \mathrm{d} s+\int_{0}^{t}(t-s)^{\alpha_{1}-1} E_{\alpha_{1}}(t-s) \sigma_{1}(s) \mathrm{d} B_{1}^{H}(s), \quad \mathbb{P}-\text { a.s., } t \in J, \\
y(t)=T_{\alpha_{2}} \phi_{2}(0)+\int_{0}^{t}(t-s)^{\alpha_{2}-1} E_{\alpha_{2}}(t-s) f_{2}\left(s, x_{s}, y_{s}\right) \mathrm{d} s+\int_{0}^{t}(t-s)^{\alpha_{2}-1} E_{\alpha_{2}}(t-s) \sigma_{2}(s) \mathrm{d} B_{2}^{H}(s), \quad \mathbb{P}-\text { a.s. }, t \in J,
\end{array}\right.
$$

where

$$
\begin{array}{ll}
T_{\alpha_{i}}(t)=\int_{0}^{\infty} \eta_{\alpha_{i}}(\theta) T_{i}\left(t^{\alpha_{i}} \theta\right) \theta, & i=1,2, \\
E_{\alpha_{i}}(t)=\alpha_{i} \int_{0}^{\infty} \theta \eta_{\alpha_{i}}(\theta) T_{i}\left(t^{\alpha_{i}} \theta\right) \theta, & i=1,2,
\end{array}
$$

in which $T(t)=e^{-t A_{i}}, t \geq 0$ is an analytic semigroup generated by the operator $-A_{i}$, and the Mainardi's Wright-type function with $\alpha_{i} \in(0,1)$ is given by

$$
\eta_{\alpha_{i}}(z)=\sum_{0}^{+\infty} \frac{(-z)^{n}}{n ! \Gamma\left(-\alpha_{i}\right) n+1-\alpha_{i}}, \quad \alpha_{i} \in(0,1), z \in \mathbb{C} .
$$

The operators $\left\{T_{\alpha_{i}}(t)\right\}_{t \geq 0}$ and $\left\{E_{\alpha_{i}}(t)\right\}_{t \geq 0}$ in (38) have the following properties [22]:

Lemma 1 (see [23]). For any $t>0$ and $\chi \in X,\left\{T_{\alpha_{i}}(t)\right\}_{t>0}$ and $\left\{E_{\alpha_{i}}(t)\right\}_{t \geq 0}$ are linear and bounded operators. Moreover, for $0<\alpha_{i}<1$ and $0 \leq v<2$, there exists a constant $C>0$ such that

$$
\begin{aligned}
& \left\|T_{\alpha_{i}}(t) \chi\right\|_{\dot{H}^{v}} \leq C t^{-\left(\alpha_{i} v / 2\right)}\|\chi\|_{X}, \\
& \left\|E_{\alpha_{i}}(t) \chi\right\|_{\dot{H}^{v}} \leq C t^{-\left(\alpha_{i} v / 2\right)}\|\chi\|_{X} .
\end{aligned}
$$

Lemma 2 (see [23]). For any $T>0$ and $\chi \in X$, the operator $E_{\alpha_{i}}(t)$ is strongly continuous. Moreover, for $0<\alpha_{i}<1$ and $0 \leq v<2$ and $0 \leq t_{1}<t_{2} \leq T$, there exists a constant $C>0$ such that

$$
\left\|\left(E_{\alpha_{i}}\left(t_{2}\right)-E_{\alpha_{i}}\left(t_{1}\right)\right) \chi\right\|_{\dot{H}^{\nu}} \leq C\left(t_{2}-t_{1}\right)^{\left(\alpha_{i} v / 2\right)}\|\chi\|_{X} .
$$

Lemma 3 (see [23]). Let $S_{\alpha_{i}}(t)=t^{\alpha_{i}-1} E_{\alpha_{i}}(t)$, for $\forall \chi \in X$, $0 \leq v<2$, and $0<\alpha_{i}<1$, there exists a constant $C>0$ such that

$$
\begin{gathered}
\left\|S_{\alpha_{i}}(t) \chi\right\|_{\dot{H}^{v}} \leq C t^{\left((2-v) \alpha_{i}-2 / 2\right)}\|\chi\|_{X}, \\
\left\|\left[S_{\alpha_{i}}\left(t_{2}\right)-S_{\alpha_{i}}\left(t_{1}\right)\right] \chi\right\|_{\dot{H}^{v}} \leq C\left(t_{2}-t_{1}\right)^{\left(2-(2-v) \alpha_{i} / 2\right)}\|\chi\|_{X} .
\end{gathered}
$$

The following lemma proves that the stochastic integral in (38) is well defined.

Lemma 4. Under the assumptions on $A, E_{\alpha_{i}}(t)$, and $\sigma(t)$, for $0 \leq v<2,0<\alpha_{i}<1$, and $1 / 4<H<1 / 2$, the stochastic integral in (38) is well defined and satisfies the following: 


$$
\mathbb{E}\left\|\int_{0}^{t}(t-s)^{\alpha_{i}-1} E_{\alpha_{i}}(t-s) \sigma_{2}(s)\right\|_{\dot{H}^{v}}^{2} \leq C t^{\delta}<\infty,
$$

where the index should satisfy

$$
\delta=\min \left\{(2-v) \alpha_{i}+4 H-3,(2-v) \alpha_{i}+2 H+\gamma-3,2 H-(2-v) \alpha_{i}+1\right\}>0 .
$$

Proof. Using the Wiener integral with respect to $\mathrm{fBm}$ and noticing the expression of $K_{t}^{*}$ and the properties of the Itô integral, for $0<H<(1 / 2)$, we get

$$
\begin{aligned}
\mathbb{E} & \int_{0}^{t}(t-s)^{\alpha_{i}-1} \mathrm{E}_{\alpha_{i}}(t-s) \sigma(s) \mathrm{d} B^{H}(s) \|_{\dot{H}^{v}}^{2} \\
= & \sum_{k=1}^{\infty} \mathbb{E}\left\|\int_{0}^{t} \lambda_{k}^{1 / 2}\left(K_{t}^{*} S_{\alpha_{i}}(t-s) e_{k} \sigma\right)(s) \mathrm{d} \beta_{k}(s)\right\|_{\dot{H}^{v}}^{2} \\
= & \sum_{k=1}^{\infty} \int_{0}^{t} \mathbb{E}\left\|\lambda_{k}^{1 / 2}\left(K_{t}^{*} S_{\alpha_{i}}(t-s) e_{k} \sigma\right)(s)\right\|_{\dot{H}^{v}}^{2} \mathrm{~d} s \\
= & \sum_{k=1}^{\infty} \int_{0}^{t} \mathbb{E} \| \lambda_{k}^{1 / 2} S_{\alpha_{i}}(t-s) e_{k} \sigma(s) K_{H}(t, s) e_{k} \\
& +\int_{s}^{t} \lambda_{k}^{1 / 2}\left[S_{\alpha_{i}}(t-r) \sigma(r)-S_{\alpha_{i}}(t-s) \sigma(s)\right] \frac{\partial K_{H}}{\partial r}(r, s) e_{k} \mathrm{~d} r \|_{\dot{H}^{\nu}}^{2} \mathrm{~d} s \\
\leq & 2 \sum_{k=1}^{\infty} \int_{0}^{t} \mathbb{E}\left\|\lambda_{k}^{1 / 2} S_{\alpha_{i}}(t-s) \sigma(s) K_{H}(t, s) e_{k}\right\|_{\dot{H}^{\nu}}^{2} \mathrm{~d} s \\
& +4 \sum_{k=1}^{\infty} \int_{0}^{t} \mathbb{E}\left\|\int_{s}^{t} \lambda_{k}^{1 / 2}\left[S_{\alpha_{i}}(t-r)(\sigma(r)-\sigma(s))\right] \frac{\partial K_{H}}{\partial r}(r, s) e_{k} \mathrm{~d} r\right\|_{\dot{H}^{\nu}}^{2} \mathrm{~d} s \\
& +4 \sum_{k=1}^{\infty} \int_{0}^{t} \mathbb{E}\left\|\int_{s}^{t} \lambda_{k}^{1 / 2} \sigma(s)\left[S_{\alpha_{i}}(t-r)-S_{\alpha_{i}}(t-s)\right] \frac{\partial K_{H}}{\partial r}(r, s) e_{k} \mathrm{~d} r\right\|_{\dot{H}^{v}}^{2} \mathrm{~d} s \\
& =I_{3} .
\end{aligned}
$$

With the help of the following inequality (see [24]):

$$
K_{H}(t, s) \leq C(H)(t-s)^{H-(1 / 2)} s^{H-(1 / 2)},
$$

furthermore, combining Lemma 3 and Hölder inequality, we obtain

$$
\begin{aligned}
I_{1} & =2 \sum_{k=1}^{\infty} \int_{0}^{t} \mathbb{E}\left\|\lambda_{k}^{1 / 2} S_{\alpha i}(t-s) \sigma(s) K_{H}(t, s) e_{k}\right\|_{\dot{H}^{v}}^{2} \mathrm{~d} s \\
& \leq 2 C(H) \widehat{\sigma}\left(\int_{0}^{t}(t-s)^{(2-v) \alpha_{i}+2 H-3 s^{2 H-1}} \sum_{k=1}^{\infty} \mathrm{E}\left\|\lambda_{k}^{1 / 2} e_{k}\right\|^{2} \mathrm{~d} s\right) \\
& \leq 2 C(H) \widehat{\sigma} \operatorname{Tr}(Q)\left(\int_{0}^{t}(t-s)^{2\left[(2-v) \alpha_{i}+2 H-3\right]} \mathrm{d} s\right)^{(1 / 2)}\left(\int_{0}^{t} s^{2(2 H-1)} \mathrm{d} s\right)^{(1 / 2)} \\
& \leq C(H, Q) \widehat{\sigma} t^{(2-v) \alpha_{i}+4 H-3},
\end{aligned}
$$


where $\hat{\sigma}:=\sup _{0 \leq s \leq T}\|\sigma(s)\|_{\mathscr{L}_{0}^{0}}<+\infty$. On the contrary, utilizing $(\mathscr{H} . .2)$, expression (17), and Hölder inequality, we get

$$
\begin{aligned}
I_{2} & =4 \sum_{k=1}^{\infty} \int_{0}^{t} \mathbb{E}\left\|\int_{s}^{t} \lambda_{k}^{1 / 2}\left[S_{\alpha_{i}}(t-r)(\sigma(r)-\sigma(s))\right] \frac{\partial K_{H}}{\partial r}(r, s) e_{k} \mathrm{~d} r\right\|_{\dot{H}^{\nu}}^{2} \mathrm{~d} s \\
& \leq 4 c_{H}^{2} C_{\sigma^{2}}^{2}-v \operatorname{Tr}(\mathrm{Q})\left(H-\frac{1}{2}\right)^{2} \int_{0}^{t} s^{1-2 H} \int_{s}^{t}\left\|\left[(t-r)^{\frac{\left(2-v \alpha_{i}-2\right.}{2}}(r-s)^{\gamma}\right](r-s)^{H-(3 / 2)} \gamma^{(1 / 2)-H}\right\|_{\dot{H}^{\nu}}^{2} \mathrm{~d} r \mathrm{~d} s \\
& \leq C(H, Q) \int_{0}^{t} s^{1-2 H}\left(\int_{s}^{t}(t-r)^{2(2-v) \alpha_{i}-4}(r-s)^{2(2 H-3+\gamma)} \mathrm{d} r\right)^{(1 / 2)}\left(\int_{s}^{t} r^{4 H-2} \mathrm{~d} r\right)^{(1 / 2)} \mathrm{d} s \\
& \leq C(H, Q) \beta\left(2(2 H-3+\gamma)+1,2(2-v) \alpha_{i}-3\right)^{(1 / 2)} \times \beta\left(2-2 H,(2-v) \alpha_{i}+4 H+\gamma-4\right) t^{(2-v) \alpha_{i}+2 H+\gamma-3},
\end{aligned}
$$

where $\beta(p, q)=\int_{0}^{1} x^{p-1}(1-x)^{q-1} \mathrm{~d} x$ is the standard Beta function, and we have used $t_{2}^{\omega}-t_{1}^{\omega} \leq C\left(t_{2}-t_{1}\right)^{\omega}$ for have

Finally, for $I_{3}$, applying Lemma 3 and expression (17), we $0 \leq \omega \leq 1$, in the above derivation.

$$
\begin{aligned}
I_{3} & =4 \sum_{k=1}^{\infty} \int_{0}^{t} \mathbb{E}\left\|\int_{s}^{t} \lambda_{k}^{1 / 2} \sigma(s)\left[S_{\alpha_{i}}(t-r)-S_{\alpha_{i}}(t-s)\right] \frac{\partial K_{H}}{\partial r}(r, s) e_{k} \mathrm{~d} r\right\|_{H^{\nu}}^{2} \mathrm{~d} s \\
& \leq 4 c_{H}^{2}\left(H-\frac{1}{2}\right)^{2} \operatorname{Tr}(Q) \widehat{\sigma}^{2} \int_{0}^{t} s^{1-2 H} \int_{0}^{t}\left\|(r-s)^{2 H-(2-v) \alpha_{i}} \gamma^{2 H-1}(r-s)^{2 H-3}\right\|_{\dot{H}^{\prime}} \mathrm{d} r \mathrm{~d} s \\
& \leq C(H, Q) \widehat{\sigma}^{2} \int_{0}^{t} s^{1-2 H}(t-s)^{4 H-(2-v) \alpha_{i}-1} \mathrm{~d} s \\
& \leq C(H, Q) \widehat{\sigma}^{2} \beta\left(2-2 H, 4 H-(2-v) \alpha_{i}\right) t^{2 H-(2-v) \alpha_{i}+1} .
\end{aligned}
$$

Then, when $\delta=\min \left\{(2-v) \alpha_{i}+4 H-3,(2-v) \alpha_{i}+2 H+\right.$ $\left.\gamma-3,2 H-(2-v) \alpha_{i}+1\right\}>0$ and $(1 / 4)<H<(1 / 2)$ and combining the above estimation inequalities of $I_{1}, I_{2}$, and $I_{3}$, we can obtain

$$
\mathbb{E}\left\|\int_{0}^{t}(t-s)^{\alpha_{i}-1} E_{\alpha_{i}}(t-s) \sigma(s) \mathrm{d} B^{H}(s)\right\|_{\dot{H}^{\nu}}^{2} \leq C t^{\delta}<\infty,
$$

where $C>0$ is a constant depending only on $H, Q, v, \gamma, \alpha_{i}$, and function $\sigma(s)$.

Theorem 2. Assume that ( $\mathscr{H} .1)-(\mathscr{H} .2)$ are satisfied and the matrix

$$
M_{\text {trice }}=\left(\begin{array}{ll}
B_{1} & B_{2} \\
B_{3} & B_{4}
\end{array}\right), \quad B_{j} \geq 0, j=1,2,3,4
$$

where

$$
\begin{aligned}
& B_{1}=\sqrt{\frac{T^{(2-v) \alpha_{1}-1} a_{f_{1}}}{(2-v) \alpha_{1}-1}}, \\
& B_{2}=\sqrt{\frac{T^{(2-v) \alpha_{1}-1} b_{f_{1}}}{(2-v) \alpha_{1}-1}}, \\
& B_{3}=\sqrt{\frac{T^{(2-v) \alpha_{2}-1} a_{f_{2}}}{(2-v) \alpha_{2}-1}}, \\
& B_{4}=\sqrt{\frac{T^{(2-v) \alpha_{2}-1} b_{f_{2}}}{(2-v) \alpha_{2}-1}} .
\end{aligned}
$$

If $M$ converges to zero, then problem (1) has a unique solution.

Proof. We consider the operator $N: \mathscr{D}_{T} \times \mathscr{D}_{T} \longrightarrow \mathscr{D}_{T} \times \mathscr{D}_{T}$ defined by

$$
N(x, y)=\left(N_{1}(x, y), N_{2}(x, y)\right),(x, y) \in \mathscr{D}_{T} \times \mathscr{D}_{T},
$$


where

$$
\begin{aligned}
& N_{1}(x, y)= \begin{cases}\phi_{1}(t), & t \in[-r, 0], \\
T_{\alpha_{1}}(t) \phi_{1}(0)+\int_{0}^{t}(t-s)^{\alpha_{1}-1} E_{\alpha_{1}}(t-s) f_{1}\left(s, x_{s}, y_{s}\right) \mathrm{d} s+\int_{0}^{t}(t-s)^{\alpha_{1}-1} E_{\alpha_{1}}(t-s) \sigma_{1}(s) \mathrm{d} B_{1}^{H}(s), & \mathbb{P}-\text { a.s, } t \in J,\end{cases}
\end{aligned}
$$

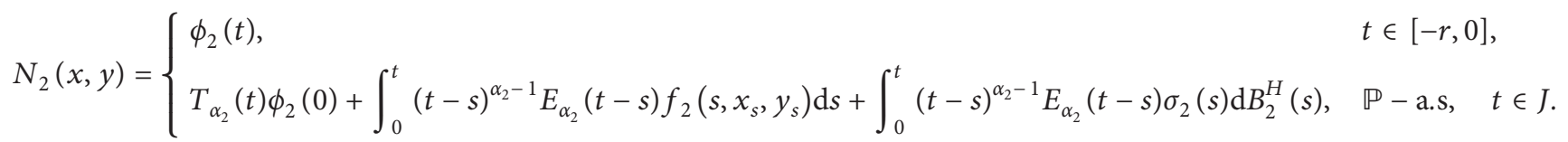

Now, we prove that $N(x, y)$ has a fixed point by Theorem 1. Indeed, let $(x, y),(\bar{x}, \bar{y}) \in \mathscr{D}_{T} \times \mathscr{D}_{T}$, and by using Lemma 1 and Hölder inequality, we obtain that

$$
\begin{aligned}
\mathbb{E} \| & N_{1}(x(t), y(t))-N_{1}(\bar{x}(t), \bar{y}(t)) \|_{\dot{H}^{v}}^{2} \\
\leq & \int_{0}^{t}(t-s)^{2 \alpha_{1}-2}\left\|S_{\alpha_{1}}(t-s)\right\|_{\dot{H}^{\prime}}^{2} \mathrm{~d} s \mathbb{E} \int_{0}^{t}\left\|\left[f_{1}\left(s, x_{s}, y_{s}\right)-f_{1}\left(s, \bar{x}_{s}, \bar{y}_{s}\right)\right]\right\|_{X}^{2} \mathrm{~d} s \\
\leq & \frac{t^{(2-v) \alpha_{1}-1} a_{f_{1}}}{(2-v) \alpha_{1}-1} \int_{0}^{t} \mathbb{E}\|x(s)-\bar{x}(s)\|_{X}^{2} \mathrm{~d} s+\frac{t^{(2-v) \alpha_{1}-1} b_{f_{1}}}{(2-v) \alpha_{1}-1} \int_{0}^{t} \mathbb{E}\|y(s)-\bar{y}(s)\|_{X}^{2} \mathrm{~d} s \\
\leq & \frac{t^{(2-v) \alpha_{1}-1} a_{f_{1}}}{(2-v) \alpha_{1}-1} \int_{0}^{t} \sup _{\tau \in J} \mathbb{E}\|x(\tau)-\bar{x}(\tau)\|_{X}^{2} \mathrm{~d} s \\
& +\frac{t^{(2-v) \alpha_{1}-1} b_{f_{1}}}{(2-v) \alpha_{1}-1} \int_{0}^{t} \sup _{\tau \in J} \mathbb{E}\|y(\tau)-\bar{y}(\tau)\|_{X}^{2} \mathrm{~d} s .
\end{aligned}
$$

Therefore, since $(x, y)=(\bar{x}, \bar{y})$ over the interval $[-r, 0]$, by taking supremum in the above inequality, we have

$$
\left\|N_{1}(x, y)-N_{1}(\bar{x}, \bar{y})\right\|_{\mathscr{D}_{T}}^{2} \leq B_{1}^{2}\|x-\bar{x}\|_{\mathscr{D}_{T}}^{2}+B_{2}^{2}\|y-\bar{y}\|_{\mathscr{D}_{T}}^{2},
$$

where

$$
\begin{aligned}
& B_{1}=\sqrt{\frac{T^{(2-v) \alpha_{1}-1} a_{f_{1}}}{(2-v) \alpha_{1}-1}}, \\
& B_{2}=\sqrt{\frac{T^{(2-v) \alpha_{1}-1} b_{f_{1}}}{(2-v) \alpha_{1}-1}} .
\end{aligned}
$$

Repeating the above process, we can also obtain

$$
\begin{aligned}
\mathbb{E} \| & N_{2}(x(t), y(t))-N_{2}(\bar{x}(t), \bar{y}(t)) \|_{\dot{H}^{v}}^{2} \\
\leq & \frac{t^{(2-v) \alpha_{2}-1} a_{f_{2}}}{(2-v) \alpha_{2}-1} \int_{0}^{t} \sup _{\tau \in J} \mathbb{E}\|x(\tau)-\bar{x}(\tau)\|_{X}^{2} \mathrm{~d} s \\
& +\frac{t^{(2-v) \alpha_{2}-1} b_{f_{2}}}{(2-v) \alpha_{2}-1} \int_{0}^{t} \sup _{\tau \in J} \mathbb{E}\|y(\tau)-\bar{y}(\tau)\|_{X}^{2} \mathrm{~d} s .
\end{aligned}
$$

$$
\left\|N_{2}(x, y)-N_{2}(\bar{x}, \bar{y})\right\|_{\mathscr{D}_{T}}^{2} \leq B_{3}^{2}\|x-\bar{x}\|_{\mathscr{D}_{T}}^{2}+B_{4}^{2}\|y-\bar{y}\|_{\mathscr{D}_{T}}^{2},
$$

where

$$
\begin{aligned}
& B_{3}=\sqrt{\frac{T^{(2-v) \alpha_{2}-1} a_{f_{2}}}{(2-v) \alpha_{2}-1}}, \\
& B_{2}=\sqrt{\frac{T^{(2-v) \alpha_{2}-1} b_{f_{2}}}{(2-v) \alpha_{2}-1}} .
\end{aligned}
$$

Hence,

$$
\begin{aligned}
\|N(x, y)-N(\bar{x}, \bar{y})\|_{\mathscr{D}_{T}} & =\left(\begin{array}{l}
\left\|N_{1}(x, y)-N_{1}(\bar{x}, \bar{y})\right\|_{\mathscr{D}_{T}} \\
\left\|N_{2}(x, y)-N_{2}(\bar{x}, \bar{y})\right\|_{\mathscr{D}_{T}}
\end{array}\right) \\
& \leq\left(\begin{array}{cc}
B_{1} & B_{2} \\
B_{3} & B_{4}
\end{array}\right)\left(\begin{array}{c}
\|x-\bar{x}\|_{\mathscr{D}_{T}} \\
\|y-\bar{y}\|_{\mathscr{D}_{T}}
\end{array}\right) .
\end{aligned}
$$

Therefore, 


$$
\begin{array}{r}
\|N(x, y)-N(\bar{x}, \bar{y})\|_{\mathscr{D}_{T}} \leq M_{\text {trice }}\left(\begin{array}{l}
\|x-\bar{x}\|_{\mathscr{D}_{T}} \\
\|y-\bar{y}\|_{\mathscr{D}_{T}}
\end{array}\right), \\
\text { for all, }(x, y),(\bar{x}, \bar{y}) \in \mathscr{D}_{T} \times \mathscr{D}_{T} .
\end{array}
$$

From Theorem 1, the mapping $N$ has a unique fixed $(x, y) \in \mathscr{D}_{T} \times \mathscr{D}_{T}$ which is a unique solution of equation (1).

Remark 1. Noticing that $B_{1}, B_{2}, B_{3}, B_{4} \in \mathbb{R}^{+}$, if $B_{1} B_{4}-B_{2} B_{3}-\left(B_{1}+B_{4}\right)>0$, then

$$
\left(\begin{array}{ll}
B_{1} & B_{2} \\
B_{3} & B_{4}
\end{array}\right)
$$

is convergent to zero.

\section{Transportation Inequalities}

In this section, we consider the property $T_{2}(C)$, for the law of the mild solution of equation (1), on the space $\mathbb{C}=C([0, T], X)$ endowed with the uniform metric $d_{\infty}$. Precisely, we have the following theorem.

Theorem 3. Assume that (H.1) and (H्H.2) holds, and let $\mathbb{P}_{\phi_{1}}, \mathbb{P}_{\phi_{2}}$ be the law of $x\left(\phi_{1}, \cdot\right), y\left(\phi_{2}, \cdot\right)$, the solution process of equation (1). Using the metric

$$
\mathrm{d}_{\infty}(x, y)=\sup _{t \in J}\|x-y\|_{X}, \quad x, y \in C([0, T], X),
$$

the probability measure $\mathbb{P}_{\phi_{1}}, \mathbb{P}_{\phi_{2}}$ satisfies " $T_{2}(C)$ " in the sense that

$$
\begin{aligned}
& {\left[W_{2}^{d_{\infty}}\left(\mathbb{Q}_{1}, \mathbb{P}_{\phi_{1}}\right)\right]^{2}+\left[W_{2}^{d_{\infty}}\left(\mathbb{Q}_{1}, \mathbb{P}_{\phi_{2}}\right)\right]^{2}} \\
& \quad \leq 2 C\left[H_{1}\left(\mathbb{Q}_{2} \mid \mathbb{P}_{\phi_{1}}\right)+H_{1}\left(\mathbb{Q}_{2} \mid \mathbb{P}_{\phi_{2}}\right)\right],
\end{aligned}
$$

on the metric space $C([0, T], X)$ with the metric $d_{\infty}$.

Proof. Let $\mathbb{P}_{\phi_{1}}, \mathbb{P}_{\phi_{2}}$ be the law of $x\left(t, \phi_{1}\right), y\left(t, \phi_{2}\right), t \in[0, T]$ on $\mathscr{C}=C[(0, T), X]$ and $\mathbb{Q}_{i}$ be any probability measure on $\mathbb{C}$ such that $\mathbb{Q}_{i} \ll \mathbb{P}_{\phi_{i}}$. Define

$$
\widetilde{\mathbb{Q}}_{i}:=\frac{\mathrm{d} \mathbb{Q}_{i}}{\mathrm{~d} \mathbb{P}_{\phi_{i}}}\left(x\left(\cdot, \phi_{i}\right)\right) \mathbb{P},
$$

which is a probability measure on $(\Omega, \mathscr{F})$. Recalling the definition of entropy and adopting a measure-transformation argument,

$$
\begin{aligned}
& H(\widetilde{\mathbb{Q}} \mid \mathbb{P})=\left(\begin{array}{l}
H_{1}\left(\tilde{\mathbb{Q}}_{2} \mid \mathbb{P}\right) \\
H_{2}\left(\widetilde{\mathbb{Q}}_{2} \mid \mathbb{P}\right)
\end{array}\right), \\
& H\left(\mathbb{Q} \mid \mathbb{P}_{\phi}\right)=\left(\begin{array}{c}
H_{1}\left(\mathbb{Q}_{1} \mid \mathbb{P}_{\phi_{1}}\right) \\
H_{2}\left(\mathbb{Q}_{2} \mid \mathbb{P}_{\phi_{2}}\right)
\end{array}\right) \\
& H_{i}\left(\widetilde{\mathbb{Q}}_{i} \mid \mathbb{P}\right)=\int_{\Omega} \log \left(\frac{\mathrm{d} \widetilde{\mathbb{Q}}_{i}}{\mathrm{~d} \mathbb{P}}\right) \mathrm{d} \widetilde{\mathbb{Q}}_{i} \\
&=\int_{\Omega} \log \left(\frac{\mathrm{d} \mathbb{Q}_{i}}{\mathrm{~d} \mathbb{P}_{\phi}}(x(\cdot, \phi))\right) \frac{\mathrm{d} \mathbb{Q}_{i}}{\mathrm{~d} \mathbb{P}_{\phi_{i}}}\left(x\left(\cdot, \phi_{i}\right)\right) \mathrm{d} \mathbb{P} \\
&=\int_{c} \log \left(\frac{\mathrm{d} \mathbb{Q}_{i}}{\mathrm{~d} \mathbb{P}_{\phi}}\right) \frac{\mathrm{d} \mathbb{Q}_{i}}{\mathrm{~d} \mathbb{P}_{\phi_{i}}} \mathrm{~d} \mathbb{P}_{\phi_{i}} \\
&=H\left(\mathbb{Q}_{i} \mid \mathbb{P}_{\phi_{i}}\right), \quad i=1,2 .
\end{aligned}
$$

Following [25], then there exists a predictable process $h_{1}(t), h_{2}(t) \in X, \quad t \in J$ with

$$
\int_{0}^{\mathrm{T}}\left\|h_{i}(s)\right\|_{X}^{2} \mathrm{~d} s<+\infty, \quad i=1,2, \mathbb{P}-\text { a.s, }
$$

such that

$$
\begin{aligned}
& \left(H_{1}\left(\mathbb{Q}_{1} \mid \mathbb{P}_{\phi_{1}}\right), H_{2}\left(\mathbb{Q}_{2} \mid \mathbb{P}_{\phi_{2}}\right)\right) \\
& \quad=\left(\frac{1}{2} \mathbb{E} \widetilde{\mathbb{Q}}_{1} \int_{0}^{\mathrm{T}}\left\|h_{1}(s)\right\|_{X}^{2} \mathrm{~d} s, \frac{1}{2} \mathbb{E}_{\widetilde{\mathbb{Q}}_{1}} \int_{0}^{\mathrm{T}}\left\|h_{2}(s)\right\|_{X}^{2} \mathrm{~d} s\right) .
\end{aligned}
$$

By the Girsanov theorem, the process $\widetilde{B}_{1}(t)$ and $\widetilde{B}_{2}(t)$ which are defined by

$$
\begin{aligned}
& \widetilde{B}_{1}(t)=B_{1}(t)-\int_{0}^{t} h_{1}(s) \mathrm{d} s, \\
& \widetilde{B}_{2}(t)=B_{1}(t)-\int_{0}^{t} h_{2}(s) \mathrm{d} s,
\end{aligned}
$$

are two Brownian motions with respect to $\left\{\mathscr{F}_{t}\right\}_{t \geq 0}$ on the probability space $\left(\Omega, \mathscr{F}, \widetilde{\mathbb{Q}}_{i}\right)$. Let us consider the $\widetilde{\mathbb{Q}}_{i}$-fractional Brownian motion $\left\{\widetilde{B}_{t}^{H}(t)\right\}_{t \in J}$ defined by

$$
\begin{aligned}
\widetilde{B}_{t}^{H}(t) & =\int_{0}^{t} K_{H}(t, s) \mathrm{d} \widetilde{B}_{i}(s) \\
& =\int_{0}^{t} K_{H}(t, s) \mathrm{d} B_{i}(s)-\left(K_{H} h_{i}\right)(t), \quad i=1,2,
\end{aligned}
$$

where $K_{H} h_{i}$ is defined by $\left(K_{H} h_{i}\right)(t)=\int_{0}^{t} K_{H}(t, s) h_{i}(s) \mathrm{d} s$. By the Fubini theorem, we obtain 


$$
\begin{aligned}
\left(K_{H} h_{i}\right)(t) & =\int_{0}^{t} c_{H}\left[\left(\frac{t}{s}\right)^{H-(1 / 2)}(t-s)^{H-(1 / 2)}-\left(H-\frac{1}{2}\right) s^{(1 / 2)-H} \int_{s}^{t} u^{H-(3 / 2)}(u-s)^{H-(1 / 2)} \mathrm{d} u\right] h_{i}(s) \mathrm{d} s \\
& =\int_{0}^{t} c_{H}\left[\left(\frac{t}{u}\right)^{H-(1 / 2)}(t-u)^{H-(1 / 2)} h_{i}(u)-\left(H-\frac{1}{2}\right) u^{H-(3 / 2)} \int_{0}^{u} u^{(1 / 2)-H}(u-s)^{H-(1 / 2)} h_{i}(s) \mathrm{d} s\right] \mathrm{d} u \\
& :=\int_{0}^{t} g_{i}(u) \mathrm{d} u, \quad i=1,2 .
\end{aligned}
$$

Consequently, under the measure $\widetilde{\mathbb{Q}}_{i}$, the process $\left\{u(t, \phi)=\left(x\left(t, \phi_{1}\right), y\left(t, \phi_{2}\right)\right)\right\}_{t \in J}$ satisfies that

$$
\begin{aligned}
& x(t)= \begin{cases}\phi_{1}(t), & t \in[-r, 0], \\
T_{\alpha_{1}}(t) \phi_{1}(0)+\int_{0}^{t}(t-s)^{\alpha_{1}-1} E_{\alpha_{1}}(t-s) f_{1}\left(s, x_{s}, y_{s}\right) \mathrm{d} s+\int_{0}^{t}(t-s)^{\alpha_{1}-1} E_{\alpha_{1}}(t-s) \sigma_{1}(s) \mathrm{d} \widetilde{B}_{1}^{H}(s) & \mathbb{P}-\text { a.s. } t \in J, \\
\quad+\int_{0}^{t}(t-s)^{\alpha_{1}-1} E_{\alpha_{1}}(t-s) \sigma_{1}(s) g_{1}(s) \mathrm{d} s, & \end{cases} \\
& y(t)=\left\{\begin{array}{lr}
\phi_{2}(t), & t \in[-r, 0], \\
T_{\alpha_{2}}(t) \phi_{2}(0)+\int_{0}^{t}(t-s)^{\alpha_{2}-1} E_{\alpha_{2}}(t-s) f_{2}\left(s, x_{s}, y_{s}\right) \mathrm{d} s+\int_{0}^{t}(t-s)^{\alpha_{2}-1} E_{\alpha_{2}}(t-s) \sigma_{2}(s) \mathrm{d} \widetilde{B}_{2}^{H}(s) & \\
\quad+\int_{0}^{t}(t-s)^{\alpha_{2}-1} E_{\alpha_{2}}(t-s) \sigma_{2}(s) g_{2}(s) \mathrm{d} s, & \mathbb{P}-\text { a.s. } t \in J .
\end{array}\right.
\end{aligned}
$$

We now consider the solution $(\bar{x}, \bar{y})$ (under $\left(\widetilde{\mathbb{Q}}_{1}, \widetilde{\mathbb{Q}}_{2}\right)$ ) of the following equation:

$$
\begin{cases}{ }^{c} D^{\alpha_{1}}(x(t))=\left(A_{1} \bar{x}(t)+f_{1}\left(t, \bar{x}_{t}, \bar{y}_{t}\right)\right) \mathrm{d} t+\sigma_{1}(t) \mathrm{d} \widetilde{B}_{1}^{H}(t), & t \in[0, T], \\ { }^{c} D^{\alpha_{2}}(y(t))=\left(A_{2} \bar{y}(t)+f_{2}\left(t, \bar{x}_{t}, \bar{y}_{t}\right)\right) \mathrm{d} t+\sigma_{2}(t) \mathrm{d} \widetilde{B}_{2}^{H}(t), & t \in[0, T], \\ x(t)=\phi_{1}(t), & t \in[-r, 0], \\ y(t)=\phi_{2}(t), & t \in[-r, 0] .\end{cases}
$$

By Theorem 2, under $\widetilde{\mathbb{Q}}_{1}, \widetilde{\mathbb{Q}}_{2}$, the law of $(x(t), \bar{x}(t)),(y(t), \bar{y}(t)), t \in[0, T]$ under $\widetilde{\mathbb{Q}}$ is a coupling of $\bar{x}\left(t, \phi_{1}\right), \bar{y}\left(t, \phi_{1}\right), t \in J \quad$ is $\quad \mathbb{P}_{\phi_{1}}, \mathbb{P}_{\phi_{2}} ; \quad$ therefore, $\quad\left(\mathbb{Q}, \mathbb{P}_{\phi}\right)$ and it follows that

$$
\left[W_{2}^{d_{\infty}}\left(\mathbb{Q}, \mathbb{P}_{\phi}\right)\right]^{2} \leq\left(\begin{array}{c}
E_{\widetilde{\mathbb{Q}}_{1}}\left(d_{\infty}(x, \bar{x})\right)^{2} \\
E_{\widetilde{\mathbb{Q}}_{2}}\left(d_{\infty}(y, \bar{y})\right)^{2}
\end{array}\right)=\left(\begin{array}{c}
E_{\widetilde{\mathbb{Q}}_{1}}\left(\sup _{t \in J}\|x(t)-\bar{x}(t)\|_{X}^{2}\right) \\
E_{\widetilde{\mathbb{Q}}_{2}}\left(\sup _{t \in J}\|y(t)-\bar{y}(t)\|_{X}^{2}\right)
\end{array}\right),
$$

where we also use the Cauchy inequality

$$
(a+b)^{2} \leq 2 a^{2}+2 b^{2} \text {. }
$$

Now, we can use the result above to estimate the distance between $u$ and $\bar{u}$ with respect to $d_{\infty}$ : 


$$
\begin{aligned}
\|x(t)-\bar{x}(t)\|_{X}^{2}= & \| \int_{0}^{t}(t-s)^{\alpha_{1}-1} E_{\alpha_{1}}(t-s)\left(f_{1}\left(s, x_{s}, y_{s}\right)\right)-\left(f_{1}\left(s, \bar{x}_{s}, \bar{y}_{s}\right)\right) \mathrm{d} s \\
& +\int_{0}^{t}(t-s)^{\alpha_{1}-1} E_{\alpha_{1}}(t-s) \sigma_{1}(s) g_{1}(s) \mathrm{d} s \|_{X}^{2} \\
\leq & \left\|\int_{0}^{t}(t-s)^{\alpha_{1}-1} E_{\alpha_{1}}(t-s)\left(f_{1}\left(s, x_{s}, y_{s}\right)\right)-\left(f_{1}\left(s, \bar{x}_{s}, \bar{y}_{s}\right)\right) \mathrm{d} s\right\|_{X}^{2} \\
& +\left\|\int_{0}^{t}(t-s)^{\alpha_{1}-1} E_{\alpha_{1}}(t-s) \sigma_{1}(s) g_{1}(s) \mathrm{d} s\right\|_{X}^{2} \\
:= & 2\left(J_{1}+J_{2}\right) .
\end{aligned}
$$

By using the Hölder inequality, condition ( $\mathscr{H} .1$ ), and Lemma 3, we obtain

$$
\begin{aligned}
J_{1} & \leq \int_{0}^{t}\left\|(t-s)^{\alpha_{1}-1} E_{\alpha_{1}}(t-s)\right\|_{\dot{H}^{\nu}}^{2} \mathrm{~d} s \int_{0}^{t}\left\|\left(f_{1}\left(s, x_{s}, y_{s}\right)-f_{1}\left(s, x_{s}, y_{s}\right)\right)\right\|_{X}^{2} \mathrm{~d} s \\
& \leq \frac{t^{(2-v) \alpha_{1}-1} a_{f_{1}}}{(2-v) \alpha_{1}-1} \int_{0}^{t}\|x(s)-\bar{x}(s)\|_{X}^{2} \mathrm{~d} s+\frac{t^{(2-v) \alpha_{1}-1} b_{f_{1}}}{(2-v) \alpha_{1}-1} \int_{0}^{t}\|y(s)-\bar{y}(s)\|_{X}^{2} \mathrm{~d} s \\
& \leq \frac{t^{(2-v) \alpha_{1}-1} a_{f_{1}}}{(2-v) \alpha_{1}-1} \int_{0}^{t} \sup _{\tau \in[0, s]}\|x(\tau)-\bar{x}(\tau)\|_{X}^{2} \mathrm{~d} s+\frac{t^{(2-v) \alpha_{1}-1} b_{f_{1}}}{(2-v) \alpha_{1}-1} \int_{0}^{t} \sup _{\tau \in[0, s]}\|y(\tau)-\bar{y}(\tau)\|_{X}^{2} \mathrm{~d} s .
\end{aligned}
$$

For the second term, using the Fubini theorem and Hölder inequality, we obtain

$$
\begin{aligned}
J_{2} \leq & 2 c_{H}^{2} \widehat{\sigma}\left\|\int_{0}^{t} S_{\alpha_{1}}(t-s)\left(\begin{array}{l}
t \\
s
\end{array}\right)^{H-(1 / 2)}(t-s)^{H-(1 / 2)} h_{1}(s) \mathrm{d} s\right\|^{2} \\
& +2\left(H-\frac{1}{2}\right) c_{H}^{2} \widehat{\sigma}\left(\left\|\int_{0}^{t} S_{\alpha_{1}}(t-s) s^{H-(3 / 2)} \int_{0}^{s} u^{(1 / 2)-H}(s-u)^{H-(1 / 2)} h_{1}(u) \mathrm{d} u \mathrm{~d} s\right\|^{2}\right) \\
\leq & 2 c_{H}^{2} \widehat{\sigma} \frac{t^{(2-v) \alpha_{1}+2 H-2}}{(2-v) \alpha_{1}+2 H-2} \int_{0}^{t}\left\|h_{1}(s)\right\|_{X}^{2} \mathrm{~d} s+4(1-H)\left(H-\frac{1}{2}\right)^{2} c_{H}^{2} \widehat{\sigma} \beta\left(2 H,(2-v) \alpha_{i}-1\right) \\
& \times \beta\left(2-2 H,(2-v) \alpha_{i}+4 H+4\right) t^{(2-v) \alpha_{i}+4 H-3} \int_{0}^{t}\left\|h_{1}(u)\right\|_{X}^{2} \mathrm{~d} u \\
:= & C_{1}^{*} \int_{0}^{t}\left\|h_{1}(s)\right\|_{X}^{2} \mathrm{~d} s .
\end{aligned}
$$

Combining (78)-(80), we have

$$
\begin{aligned}
\|x(s)-\bar{x}(s)\|_{X}^{2} \leq & \frac{t^{(2-v) \alpha_{1}-1} a_{f_{1}}}{(2-v) \alpha_{1}-1} \int_{0}^{t} \sup _{\tau \in J}\|x(\tau)-\bar{x}(\tau)\|_{X}^{2} \mathrm{~d} s \\
& +\frac{t^{(2-v) \alpha_{1}-1} b_{f_{1}}}{(2-v) \alpha_{1}-1} \int_{0}^{t} \sup _{\tau \in J}\|y(\tau)-\bar{y}(\tau)\|_{X}^{2} \mathrm{~d} s \\
& +C_{1}^{*} \int_{0}^{t}\left\|h_{1}(s)\right\|_{X}^{2} \mathrm{~d} s .
\end{aligned}
$$


Similarly, we have

$$
\begin{aligned}
\|y(s)-\bar{y}(s)\|_{X}^{2} \leq & \frac{t^{(2-v) \alpha_{1}-1} a_{f_{2}}}{(2-v) \alpha_{2}-1} \int_{0}^{t} \sup _{\tau \in J}\|x(\tau)-\bar{x}(\tau)\|_{X}^{2} \mathrm{~d} s \\
& +\frac{t^{(2-v) \alpha_{1}-1} b_{f_{2}}}{(2-v) \alpha_{2}-1} \int_{0}^{t} \sup _{\tau \in J}\|y(\tau)-\bar{y}(\tau)\|_{X}^{2} \mathrm{~d} s \\
& +C_{2}^{*} \int_{0}^{t}\left\|h_{2}(s)\right\|_{X}^{2} \mathrm{~d} s .
\end{aligned}
$$

Adding (81) and (82), we obtain

$$
\begin{aligned}
& \sup _{s \in J}\left(\|x(s)-\bar{x}(s)\|_{X}^{2}+\|y(s)-\bar{y}(s)\|_{X}^{2}\right) \\
& \leq \widehat{C} \int_{0}^{t} \sup _{\tau \in[0, s]}\left(\|x(\tau)-\bar{x}(\tau)\|_{X}^{2}+\|y(\tau)-\bar{y}(\tau)\|_{X}^{2}\right) \mathrm{d} s \\
& \quad+C^{*} \int_{0}^{t}\left\|h_{1}(s)\right\|_{X}^{2}+\left\|h_{2}(s)\right\|_{X}^{2} \mathrm{~d} s,
\end{aligned}
$$

where $\quad \widehat{C}=\max \left\{2\left(t^{(2-v) \alpha_{1}-1}\left(a_{f_{1}}+a_{f_{2}}\right) /(2-v) \alpha_{1}-1\right)\right.$, $\left.2\left(t^{(2-v) \alpha_{1}-1}\left(b_{f_{1}}+b_{f_{2}}\right) /(2-v) \alpha_{1}-1\right)\right\}$ and $C^{*}=\max \left\{C_{1}^{*}\right.$, $\left.\mathrm{C}_{2}^{*}\right\}$. By using the Gronwall inequality, we have

$$
\begin{aligned}
& \sup _{s \in[0, t]}\left(\|x(s)-\bar{x}(s)\|_{X}^{2}+\|y(s)-\bar{y}(s)\|_{X}^{2}\right) \\
& \quad \leq C^{*} \exp (\widehat{C} T) \int_{0}^{T}\left\|h_{1}(s)\right\|_{X}^{2}+\left\|h_{2}(s)\right\|_{X}^{2} \mathrm{~d} s .
\end{aligned}
$$

Hence, it follows that

$$
\begin{aligned}
& {\left[W_{2}^{d_{\infty}}\left(\mathbb{Q}_{1}, \mathrm{P}_{\phi_{1}}\right)\right]^{2}+\left[W_{2}^{d_{\infty}}\left(\mathbb{Q}_{2}, \mathrm{P}_{\phi_{2}}\right)\right]^{2}} \\
& \quad \leq C^{*} \exp (\widehat{C} T) E_{\widetilde{Q}_{1}}\left(\int_{0}^{T}\left\|h_{1}(s)\right\|_{X}^{2}+\left\|h_{2}(s)\right\|_{X}^{2} \mathrm{~d} s\right) \\
& \quad \leq 2 C\left[H_{1}\left(\mathbb{Q}_{1} \mid p_{\phi_{1}}\right)+H_{2}\left(\mathbb{Q}_{2} \mid p_{\phi_{2}}\right)\right],
\end{aligned}
$$

where $C=C^{*} \exp (\widehat{C} T)$. The proof is complete.

Remark 2. In [15], by using the Girsanov theorem for fractional Brownian motion, the authors established the transportation inequalities for the law of the mild solution to stochastic evolution equations driven by the fractional Brownian motion with the Hurst parameter $H \in((1 / 2), 1)$. Besides, Boufoussi and Hajji [16] established the transportation inequalities for the law of the mild solution to stochastic evolution equations driven by the fractional Brownian motion with the Hurst parameter $H \in(0,(1 / 2))$. However, the transportation inequalities for fractional stochastic evolution equations driven by the fractional Brownian motion are more complicated. On the contrary, for a coupled system, we have to consider the transportation inequalities for the law of the random vector $(X(t), Y(t))$, which is more difficult. So, our results generalize and improve the results in $[15,16]$.

\section{An Example}

In this section, we present an example to illustrate the usefulness and applicability of our results. We consider the following fractional stochastic partial differential equation with delay effects:

$$
\begin{cases}{ }^{c} D^{\alpha_{1}}(u(t, x))=\frac{\partial^{2}}{\partial x^{2}} u(t, x)+\left(1-a_{1} u(t, x(t-\tau))(\sin t+\sin (\sqrt{2} t))\right) & t \in[0, T], 0 \leq x \leq \pi, \\ -b_{1} v\left(t, x(t-\tau)(\cos t+\cos (\sqrt{2 t}))+e^{-t\left(\mathrm{~d} B^{H} / \mathrm{d} t\right),}\right. & t \in[0, T], 0 \leq x \leq \pi, \\ { }^{c} D^{\alpha_{1}}(u(t, x))=\frac{\partial^{2}}{\partial x^{2}} u(t, x)+\left(1-a_{2} u(t, x(t-\tau))(\sin t+\sin (\sqrt{2} t))\right) & t \in[0, T], \\ -b_{2} v((t, x(t-\tau))(\cos t+\cos (\sqrt{2 t})))+e^{-t\left(\mathrm{~d} B^{H} / \mathrm{d} t\right),} & t \in[0, T], \\ u(t, 0)=u(t, \pi)=0, & t \in[-r, 0], 0 \leq x \leq \pi, \\ v(t, 0)=v(t, \pi)=0, & t \in[-r, 0], 0 \leq x \leq \pi, \\ u(t, x)=\phi_{1}(t, x), & t \\ v(t, x)=\phi_{2}(t, x), & t\end{cases}
$$


where $a_{i}, b_{i}>0, \alpha_{i} \in((2 / 3), 1]$ and $\tau>0, B^{H}$ denotes a fractional Brownian motion. To rewrite this system into the abstract form (1), we set

$$
\begin{aligned}
f_{1}\left(t, \phi_{1 t}, \phi_{2 t}\right)(\eta)= & 1-a_{1}\left(\phi_{1}\left(\eta_{t}\right)(\sin t+\sin (\sqrt{2} t))\right) \\
& -b_{1}\left(\phi_{2}\left(\eta_{t}\right)(\cos t+\cos (\sqrt{2 t}))\right), \\
f_{2}\left(t, \phi_{1 t}, \phi_{2 t}\right)(\eta)= & 1-a_{2}\left(\phi_{1}\left(\eta_{t}\right)(\sin t+\sin (\sqrt{2} t))\right) \\
& -b_{2}\left(\phi_{1}\left(\eta_{t}\right)(\cos t+\cos (\sqrt{2} t))\right), \\
\sigma_{1}(t)= & e^{-t}, \\
\sigma_{2}(t)= & 2 e^{-t},
\end{aligned}
$$

and $\mathscr{K}=\mathscr{H}=L^{2}([0, \pi])$. We denote the operator $A$ by $A u=u^{\prime \prime}$, with domain $D(A)=\left\{u \in \mathscr{H}, u^{\prime \prime} \in \mathscr{H}\right.$ and $u(0)=u(\pi)=0\}$.

Then, it is easy to obtain

$$
A z=-\sum_{n=1}^{\infty} e^{-n^{2} t}\left\langle z, e_{n}\right\rangle e_{n}, \quad z \in \mathscr{H}
$$

and $A$ is the infinitesimal generator of an analytic semigroup $\{S(t)\}_{t \geq 0}$ on $\mathscr{H}$, which has following the following formula:

$$
S(t) u=\sum_{n=1}^{\infty} e^{-n^{2} t}\left\langle u, e_{n}\right\rangle e_{n}, \quad u \in \mathscr{H},
$$

where $e_{n}(u)=(2 / \pi)^{(1 / 2)} \sin (n u), n=1,2, \ldots$, is the orthogonal set of eigenvectors of $A$. If the analytic semigroup $\{S(t)\}, t \in J$, is compact, then there exists a constant $K \geq 1$ such that $\|S(t)\|^{2} \leq K$.

In order to define the operator $Q: \mathscr{K} \longrightarrow \mathscr{K}$, we choose a sequence $\left\{Q e_{n}=\sigma_{n} e_{n}\right\}$ and assume that

$$
\operatorname{tr}(Q)=\sum_{n=1}^{\infty} \sqrt{\sigma_{n}}<\infty \text {. }
$$

Define the process $B^{H}(s)$ by

$$
B^{H}=\sum_{n=1}^{\infty} \sqrt{\sigma_{n}} B_{n}^{H}(t) e_{n}
$$

where $1 / 4<H<1 / 2$ and $\left\{B_{n}^{H}\right\}_{n \in \mathbb{N}}$ is a sequence of two-sided one-dimensional mutually independent fractional Brownian motions. Thus, one has

$$
\begin{aligned}
& \left\|f_{1}(t, x, y)-f_{2}(t, \bar{x}, \bar{y})\right\|^{2} \leq 8 a_{1}\|x-\bar{x}\|_{\mathscr{D}_{0}}+8 b_{1}\|y-\bar{y}\|_{\mathscr{D}_{0}}, \\
& \left\|f_{2}(t, x, y)-f_{2}(t, \bar{x}, \bar{y})\right\|^{2} \leq 8 a_{2}\|x-\bar{x}\|_{\mathscr{D}_{0}}+8 b_{2}\|y-\bar{y}\|_{\mathscr{D}_{0}} .
\end{aligned}
$$

On account of the conditions, it is straightforward to check that $(\mathscr{H} .1)$ and $(\mathscr{H} .2)$ hold. Let

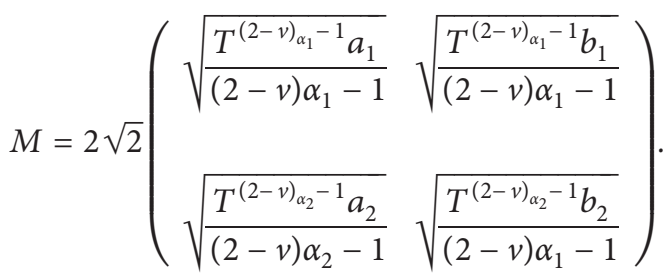

If $M$ converges to zero, then assumptions in Theorem 2 are fulfilled, then we can conclude that the law of the unique mild solution of system (86) on $[0, T]$ satisfies the property $T_{2}(C)$.

\section{Conclusion}

In this paper, by Perov's fixed-point theorem, some stochastic analysis technique, and the properties of operator semigroup, we show the existence and uniqueness of the mild solution for a class of coupled fractional stochastic evolution equations driven by the fractional Brownian motion with the Hurst parameter $H \in(1 / 4,1 / 2)$. Furthermore, we establish the transportation inequalities for the law of the mild solution, with respect to the uniform distance. In our next paper, we will explore the existence, uniqueness, and the transportation inequalities of the mild solution for a class of coupled fractional stochastic evolution equations driven by the fractional Brownian motion with the Hurst parameter $H \in(0,1 / 4)$.

\section{Data Availability}

The data used to support the findings of the study are available from the corresponding author upon request.

\section{Conflicts of Interest}

The authors declare that they have no conflicts of interest.

\section{Authors' Contributions}

All authors contributed equally to this work and read and approved the final manuscript.

\section{Acknowledgments}

This research was partially supported by the NNSF of China (no. 11571071), the Natural Science Foundation of Hubei Province (no. 2016CFB479), and the cultivation plan of excellent doctoral and master's dissertations in Yangtze University (no. YS2018038).

\section{References}

[1] K. Balachandran, S. Kiruthika, and J. J. Trujillo, "On fractional impulsive equations of Sobolev type with nonlocal condition in Banach spaces," Computers \& Mathematics with Applications, vol. 62, no. 3, pp. 1157-1165, 2011.

[2] Podlubny, Fractional Differential Equations, Academic Press, San Diego, CA, USA, 1999.

[3] A. A. Kilbas, H. M. Srivastava, and J. J. Trujillo, Theory and Applications of Fractional Differential Equations, Elsevier. Science Limited, Amsterdam, Netherlands, 2006.

[4] X. Zhang, P. Chen, A. Abdelmonem, and Y. Li, "Fractional stochastic evolution equations with nonlocal initial conditions and noncompact semigroups," Stochastics, vol. 90, no. 7, pp. 1005-1022, 2018.

[5] V. V. Anh and W. Grecksch, "A fractional stochastic evolution equation driven by fractional Brownian motion," Monte Carlo Methods and Applications MCMA, vol. 9, no. 3, pp. 189-199, 2003. 
[6] J. Luo and K. Liu, "Stability of infinite dimensional stochastic evolution equations with memory and Markovian jumps," Stochastic Processes and Their Applications, vol. 118, no. 5, pp. 864-895, 2008.

[7] R. Sakthivel, P. Revathi, and Y. Ren, "Existence of solutions for nonlinear fractional stochastic differential equations," Nonlinear Analysis: Theory, Methods \& Applications, vol. 81, pp. 70-86, 2013.

[8] K. Li, "Stochastic delay fractional evolution equations driven by fractional Brownian motion," Mathematical Methods in the Applied Sciences, vol. 38, no. 8, pp. 1582-1591, 2015.

[9] G. M. Mophou, "Existence and uniqueness of mild solutions to impulsive fractional differential equations," Nonlinear Analysis: Theory, Methods \& Applications, vol. 72, no. 3-4, pp. 1604-1615, 2010.

[10] M. Talagrand, "Transportation cost for Gaussian and other product measures," Geometric and Functional Analysis, vol. 6, no. 3, pp. 587-600, 1996.

[11] L. Wu and Z. Zhang, "Talagrand's T2-transportation inequality and log-sobolev inequality for dissipative SPDEs and applications to reaction-diffusion equations," Chinese Annals of Mathematics, Series B, vol. 27, no. 3, pp. 243-262, 2006.

[12] A. S. Üstünel, “Transportation cost inequalities for diffusions under uniform distance," Springer Proceedings in Mathematics \& Statistics, vol. 22, pp. 203-214, 2012.

[13] J. Bao, F.-Y. Wang, and C. Yuan, "Transportation cost inequalities for neutral functional stochastic equations," Zeitschrift für Analysis und ihre Anwendungen, vol. 32, no. 4, pp. 457-475, 2013.

[14] B. Saussereau, "Transportation inequalities for stochastic differential equations driven by a fractional Brownian motion," Bernoulli, vol. 18, no. 1, pp. 1-23, 2012.

[15] Z. Li and J. Luo, "Transportation inequalities for stochastic delay evolution equations driven by fractional Brownian motion," Frontiers of Mathematics in China, vol. 10, no. 2, pp. 303-321, 2015.

[16] B. Boufoussi and S. Hajji, "Transportation inequalities for neutral stochastic differential equations driven by fractional brownian motion with hurst parameter lesser than 1/2," Mediterranean Journal of Mathematics, vol. 14, pp. 192-208, 2017.

[17] F. Biagini, Y. Hu, B. Oksendal, and T. Zhang, Stochastic Calculus for Fractional Brownian Motion and Applications, Springer, Berlin, Germany, 2008.

[18] D. Nualart, The Malliavin Calculus and Related Topics, Springer, Berlin, Germany, 2006.

[19] A. I. Perov, "On the Cauchy problem for a system of ordinary differential equations Pviblizhen," Pviblizhen. Met. Reshen. Differ. Uvavn, vol. 2, pp. 115-134, 1964, in Russian.

[20] R. Precup, Methods in Nonlinear Integral Equations, Springer Science, Berlin, Germany, 2013.

[21] R. Precup, "The role of matrices that are convergent to zero in the study of semilinear operator systems," Mathematical and Computer Modelling, vol. 49, no. 3-4, pp. 703-708, 2009.

[22] G.-a. Zou and B. Wang, "Stochastic Burgers' equation with fractional derivative driven by multiplicative noise," Computers \& Mathematics with Applications, vol. 74, no. 12, pp. 3195-3208, 2017.

[23] G.-a. Zou, G. Lv, and J.-L. Wu, "Stochastic Navier-Stokes equations with Caputo derivative driven by fractional noises," Journal of Mathematical Analysis and Applications, vol. 461, no. 1, pp. 595-609, 2018.

[24] G. Wang, M. Zeng, and B. Guo, "Stochastic Burgers' equation driven by fractional Brownian motion," Journal of Mathematical Analysis and Applications, vol. 371, no. 1, pp. 210-222, 2010.
[25] H. Djellout, A. Guillin, and L. Wu, "Transportation costinformation inequalities and applications to random dynamical systems and diffusions," The Annals of Probability, vol. 32, no. 3B, pp. 2702-2732, 2004. 